\section{OPEN ACCESS}

Edited by:

Zisis Kozlakidis, International Agency for Research on

Cancer (IARC), France

Reviewed by:

Xianbo Wang

Capital Medical University, China

Rajat Kapoor

Indiana University, United States Ahmed Said,

Washington University in St. Louis,

United States

*Correspondence: Zhongij Meng

zhongji.meng@163.com

Jun Zhao

stzhao@163.com

†These authors have contributed equally to this work

Specialty section:

This article was submitted to Infectious Diseases - Surveillance,

Prevention and Treatment,

a section of the journa

Frontiers in Medicine

Received: 22 September 2020

Accepted: 14 January 2021

Published: 18 February 2021

Citation:

Chen Y, Zhou X, Yan H, Huang H, Li S, Jiang $Z$, Zhao $J$ and Meng $Z$ (2021) CANPT Score: A Tool to Predict Severe COVID-19 on Admission. Front. Med. 8:608107. doi: 10.3389/fmed.2021.608107

\title{
CANPT Score: A Tool to Predict Severe COVID-19 on Admission
}

\author{
Yuanyuan Chen ${ }^{1,2+}$, Xiaolin $\mathrm{Zhou}^{3 \dagger}$, Huadong Yan ${ }^{4 \dagger}$, Huihong Huang ${ }^{5+}$, Shengjun $\mathrm{Li}^{1}$, \\ Zicheng Jiang ${ }^{5}$, Jun Zhao ${ }^{1,6 *}$ and Zhongji Meng ${ }^{1,2,7 *}$
}

${ }^{1}$ Department of Infectious Diseases, Taihe Hospital, Hubei University of Medicine, Shiyan, China, ${ }^{2}$ Institute of Biomedical Research, Taihe Hospital, Hubei University of Medicine, Shiyan, China, ${ }^{3}$ Department of Liver Diseases, Yichang Central People's Hospital, China Three Gorges University, Yichang, China, ${ }^{4}$ Department of Liver Diseases, HwaMei Hospital, University of Chinese Academy of Sciences, Ningbo, China, ${ }^{5}$ Department of Infectious Diseases, Ankang Central Hospital, Hubei University of Medicine, Ankang, China, ${ }^{6}$ School of Public Health, Hubei University of Medicine, Shiyan, China, ${ }^{7}$ Hubei Clinical Research Center for Precise Diagnosis and Treatment of Liver Cancer, Shiyan, China

Background and Aims: Patients with critical coronavirus disease 2019 (COVID-19) have a mortality rate higher than $50 \%$. The purpose of this study was to establish a model for the prediction of the risk of severe disease and/or death in patients with COVID-19 on admission.

Materials and Methods: Patients diagnosed with COVID-19 in four hospitals in China from January 22, 2020 to April 15, 2020 were retrospectively enrolled. The demographic, laboratory, and clinical data of the patients with COVID-19 were collected. The independent risk factors related to the severity of and death due to COVID-19 were identified with a multivariate logistic regression; a nomogram and prediction model were established. The area under the receiver operating characteristic curve (AUROC) and predictive accuracy were used to evaluate the model's effectiveness.

Results: In total, 582 patients with COVID-19, including 116 patients with severe disease, were enrolled. Their comorbidities, body temperature, neutrophil-to-lymphocyte ratio (NLR), platelet $(\mathrm{PLT})$ count, and levels of total bilirubin (Tbil), creatinine (Cr), creatine kinase (CK), and albumin (Alb) were independent risk factors for severe disease. A nomogram was generated based on these eight variables with a predictive accuracy of $85.9 \%$ and an AUROC of $0.858(95 \% \mathrm{Cl}, 0.823-0.893)$. Based on the nomogram, the CANPT score was established with cut-off values of 12 and 16. The percentages of patients with severe disease in the groups with CANPT scores $<12, \geq 12$, and $<16$, and $\geq 16$ were 4.15, 27.43, and 69.64\%, respectively. Seventeen patients died. NLR, Cr, CK, and Alb were independent risk factors for mortality, and the CAN score was established to predict mortality. With a cut-off value of 15 , the predictive accuracy was $97.4 \%$, and the AUROC was 0.903 (95\% Cl 0.832, 0.974).

Conclusions: The CANPT and CAN scores can predict the risk of severe disease and mortality in COVID-19 patients on admission.

Keywords: SARS-CoV-2, COVID-19, severe illness, prediction, nomogram 


\section{INTRODUCTION}

Coronavirus disease 2019 (COVID-19) is caused by severe respiratory syndrome coronavirus 2 (SARS-CoV-2), and pneumonia is the main clinical manifestation (1-3). SARS-CoV2 is highly transmissible $(4,5)$, and the COVID-19 pandemic has spread to every country. With the rapid increase in the number of confirmed cases, medical resources have been inadequate (6).

As of December 30, 2020, the cumulative number of confirmed cases worldwide exceeded 80 million, and more than 1.78 million patients had died; the daily number of newly diagnosed patients is still rising. Although many clinical trials have been performed in the treatment of COVID-19 patients, so far, only dexamethasone has been validated in reducing the mortality rate of critically ill patients, and no specific medicine is available for COVID-19 (7). The demand for intensive care unit (ICU) beds, ventilators, protective equipment, other medical resources and medical staff exceed the existing supply by 10 -fold (6). The early identification of patients at risk for severe disease and death, the timely initiation of interventions and admission to the ICU can prevent disease progression and reduce the mortality rate. Patients with mild COVID-19 require access to only limited medical resources for isolation and general symptomatic treatment. Therefore, it is very important to establish models predicting the prognoses of patients with COVID-19. More than 700 prognosis-related articles have been published in journals and on preprint platforms; most articles have only provided the risk factors for a poor outcome in COVID-19 patients; and $\sim 50$ prognostic models have been reported (8). Yuan et al. reported a model with good predictive efficacy [area under the receiver operating characteristic curve $($ AUROC) $=0.901]$ in predicting the risk of mortality in patients with COVID-19 using chest computed tomography (CT) scores. However, this model is not sufficiently representative and generalizable because only 27 patients from Wuhan were included (9). Other models were constructed using data only from patients in Wuhan (9-13), and many models have been presented as nomograms, which are inconvenient for clinical application and have not been verified in populations of other COVID-19 patients $(12,14-16)$.

Liang et al. established a model based on 1,590 COVID-19 patients from 31 provinces in China and validated this model in another 710 patients with COVID-19; the model is available on a web page (http://118.126.104.170/). The model showed good predictive ability for a poor prognosis of COVID-19 in both the development cohort and the external validation cohort (AUROC $=0.880$ ). The following 10 variables were included the model: X-ray abnormalities, age, hemoptysis, dyspnea, unconsciousness, number of comorbidities, cancer history, neutrophil-to-lymphocyte ratio (NLR), lactate dehydrogenase (LDH) level, and direct bilirubin level; however, only the NLR, LDH level, and direct bilirubin level are biochemical indicators, which are inadequate for enabling a comprehensive evaluation of renal, heart, and coagulation function in patients with COVID19. This model and several other models used ICU admission, invasive ventilation, and death as the composite outcome (14, 1618 ), although not all patients with critical disease require ICU admission and/or invasive ventilation, and more than $50 \%$ of patients with critical disease will die (6); thus, individual models are needed for the precise prediction of severe disease or death. $\mathrm{Wu}$ et al. reported a model with good predictive efficacy (16) that was established based on data from 299 patients from Wuhan, China, and verified in 426 patients with COVID-19 from China, Italy, and Belgium. However, Collins et al. believed that the sample size of patients in their study was relatively small and that it was unreasonable to use 239 patients for model development and 60 patients for internal validation. The patients with a predicted risk of a poor prognosis from 21.00 to $80.00 \%$ were classified into the medium-risk group, further casting doubt regarding the basis of risk stratification in the study (19). The CALL score developed by Ji et al. includes comorbidities, lymphocytes, age, and LDH, has good predictive efficacy and is convenient for clinical use. However, the sample size in their study was relatively small, and there was no external validation (20). The NLR has been reported to be a prognostic factor in COVID-19 $(21,22)$; however, the NLR can only reflect the status of the immune system and is insufficient for assessing the comprehensive situation in COVID-19 patients because COVID19 is a systemic disease $(23,24)$. In summary, these models have a risk of bias, and their reliability in clinical application has not been verified $(8,25)$. As the COVID-19 epidemic continues to spread, it is necessary to develop a reliable and clinically applicable prognostic model for COVID-19.

In this study, by comparing the demographic, clinical, and blood biochemical characteristics of COVID-19 patients with and without severe disease on admission, the risk factors for severe disease and mortality were identified, and risk prediction models for severe disease and death in COVID-19 patients were established.

\section{MATERIALS AND METHODS}

\section{Patient Selection}

This study retrospectively included patients with COVID-19 diagnosed at Shiyan Taihe Hospital, Ankang Central Hospital, Ningbo Hwamei Hospital, and Yichang Central People's Hospital from January 22, 2020 to April 15, 2020. The criteria used for the diagnosis and classification of confirmed cases of COVID19 were provided in the "Guidance for 2019 coronavirus disease prevention, control, diagnosis and management" (26). The clinical classifications were as follow. (1) Mild: the clinical symptoms were mild, and no pneumonia manifestations were observed on imaging. (2) Moderate: patients had symptoms, such as fever and respiratory tract symptoms, and pneumonia manifestations were observed on imaging. (3) Severe: any of the following criteria were met: (1) respiratory distress, respiration rate $(\mathrm{RR}) \geq 30$ breaths/min; (2) pulse oxygen saturation ( $\mathrm{SpO} 2$ ) $\leq 93 \%$ on room air at rest; or (3) arterial partial pressure of oxygen $(\mathrm{PaO} 2)$ /oxygen concentration $(\mathrm{FiO} 2) \leq 300 \mathrm{mmHg}$. In regions with a high altitude (more than 1 kilometer above sea level), the $\mathrm{PaO} 2 / \mathrm{FiO} 2$ values were adjusted based on the following: equation of $\mathrm{PaO} 2 / \mathrm{FiO} 2 \times$ [atmospheric pressure $(\mathrm{mm} \mathrm{Hg}) / 760$ ]. Patients with $>50 \%$ lesion progression within 24 to $48 \mathrm{~h}$ on pulmonary imaging were treated as having severe cases. And (4) Critical: any of the following criteria were met: (1) respiratory failure 
needing mechanical ventilation; (2) shock; or (3) other organ failure requiring monitoring and treatment in the ICU. In this study, the severe and critical cases were classified as having severe disease, while the mild and moderate cases were classified as having non-severe disease. Patients diagnosed with severe disease on admission were only included in the mortality risk analysis. This study was approved by the Medical Ethics Committee of Shiyan Taihe Hospital. The approval number is 2020KS018.

\section{Data Collection}

Clinical data pertaining to COVID-19 patients on admission were retrieved from the medical record databases of Shiyan Taihe Hospital, Ankang Central Hospital, Ningbo Hwamei Hospital, and Yichang Central People's Hospital. The data included the patients' epidemiological histories, comorbidities, vital signs (heart rate, RR, blood pressure, and body temperature), signs and symptoms (fever), laboratory tests (liver and kidney function, routine blood tests, C-reactive protein (CRP) levels, and chest CT findings), and outcome at discharge. The patients with COVID-19 who progressed to severe or critical disease during hospitalization were included in the analysis of severe disease. Survival at discharge was the final outcome of this study. The included comorbidities were hypertension, diabetes, cardiocerebrovascular disease, malignant tumor, chronic liver disease, chronic kidney disease, and chronic lung disease.

\section{Statistical Analysis}

The normally and non-normally distributed continuous variables are presented as the means \pm standard deviations (SDs) and medians (interquartile ranges, IQRs), respectively. The categorical variables are presented as $n(\%) . t$-tests, chi-square tests and Mann-Whitney $U$-tests were used to compare the differences in various indicators between the two groups. To ensure that the variables conformed to a normal distribution to the greatest extent possible, natural logarithmic transformation was applied to the white blood cell (WBC) count, procalcitonin (PCT) level, CRP level and other variables. Then, we obtained the natural logarithm (ln) of WBC [ln (WBC)], ln (NLR), ln (PLT), ln (Alb), ln (Tbil), ln (Cr), etc. During the modeling process, variables with more than $10 \%$ missing values were excluded from the analysis, and variables with $<10 \%$ missing values were addressed with multiple imputation. The independent prognostic risk factors were selected by a logistic regression analysis and included in the nomogram, which was used to establish the prediction model. For convenience in clinical application, the independent risk factors identified by the logistic regression analysis were converted into dichotomous variables with a cutoff value determined by receiver operating characteristic (ROC) curve analysis. A logistic regression was performed to determine the weight of the influence of the variables on disease progression and establish a new scoring model. The best cut-off value was determined according to the Youden index, sensitivity, specificity, predictive value, and likelihood ratio. The leave-oneout cross validation method was used for internal validation, and 1,000 bootstrap resamplings were performed. The AUROC and Hosmer-Lemeshow test were used to evaluate the predictive efficacy of the model. SPSS software, version 22.0 (SPSS, Inc.,
Chicago, IL, USA), was used for the data analysis. The nomogram was established using R software, version 3.6.1 (R Foundation for Statistical Computing, Vienna, Austria). A two-tailed $P<0.05$ was considered statistically significant.

\section{RESULTS}

\section{Baseline Characteristics of COVID-19 Patients}

In total, 582 patients with COVID-19, including 202 from Shiyan Taihe Hospital, 40 from Ankang Central Hospital, 108 from Ningbo Hwamei Hospital, and 232 from Yichang Central People's Hospital, were enrolled in this study. During hospitalization, 116 patients developed severe disease, and 17 patients died. There were 466 patients with non-severe COVID-19, including 25 with mild cases and 441 with moderate cases (Figure 1). The median age of the patients with severe disease was significantly higher than that of the patients with non-severe disease (63.00 vs. 47.00, $P<0.001)$. The proportion of patients with comorbidities in the severe disease group was almost two times greater than that in the non-severe disease group (59.48 vs. $24.68 \%, P<0.001)$. Hypertension was the most common comorbidity in the patients with COVID-19 (20.10\%), followed by diabetes (9.28\%) and malignant tumors $(2.23 \%)$, which were more common in the patients with severe disease than those with non-severe disease (Table 1). Among the 582 patients with COVID-19, fever was the most common symptom (74.05\%), and the incidence of fever in the patients with severe disease was higher than that in the patients with non-severe disease, although the difference was not statistically significant ( 80.17 vs. $72.53 \%, P=0.093)$. However, the proportion of patients with a body temperature $\geq 38.5^{\circ} \mathrm{C}$ in the group with severe disease was significantly higher than that in the group with non-severe disease (41.38 vs. $22.32 \%, P<0.001)$; intrapulmonary ground-glass opacities (GGOs) were observed on CT in 559 /582 (96.05\%) patients with COVID-19, including all patients with severe disease, and 443/466 (95.06\%) patients with non-severe disease. There was no significant difference between the two groups in terms of sex, respiration, heart rate, or blood pressure on admission (Table 1).

The lymphocyte (LY) count was reduced in 300/582 (51.55\%) patients with COVID-19, and a reduced LY count was more common in the group with severe disease than in the group with non-severe disease (76.72 vs. $45.92 \%, P<0.001)$. However, the group with severe disease had a significantly larger proportion of patients with elevated WBC counts, especially neutrophils (NE), than the group with non-severe disease (11.21 vs. $3.22 \%, P<$ $0.001 ; 23.28$ vs. $7.73 \%, P<0.001)$. Therefore, the patients with severe disease had a significantly higher NLR than those with non-severe disease $(4.20$ vs. 2.64, $P<0.001)$. The proportions of patients with reduced hemoglobin (HGB) and PLT levels were two times higher in the group with severe disease than in the group with non-severe disease (14.66 vs. $7.30 \%, P=0.012$ 29.31 vs. $13.52 \%, P<0.001)$. The rates of abnormal aspartate aminotransferase (AST) and $\gamma$-glutamyl transpeptidase (GGT) levels were significantly higher in the patients with severe disease than in the patients with non-severe disease. A reduced albumin 


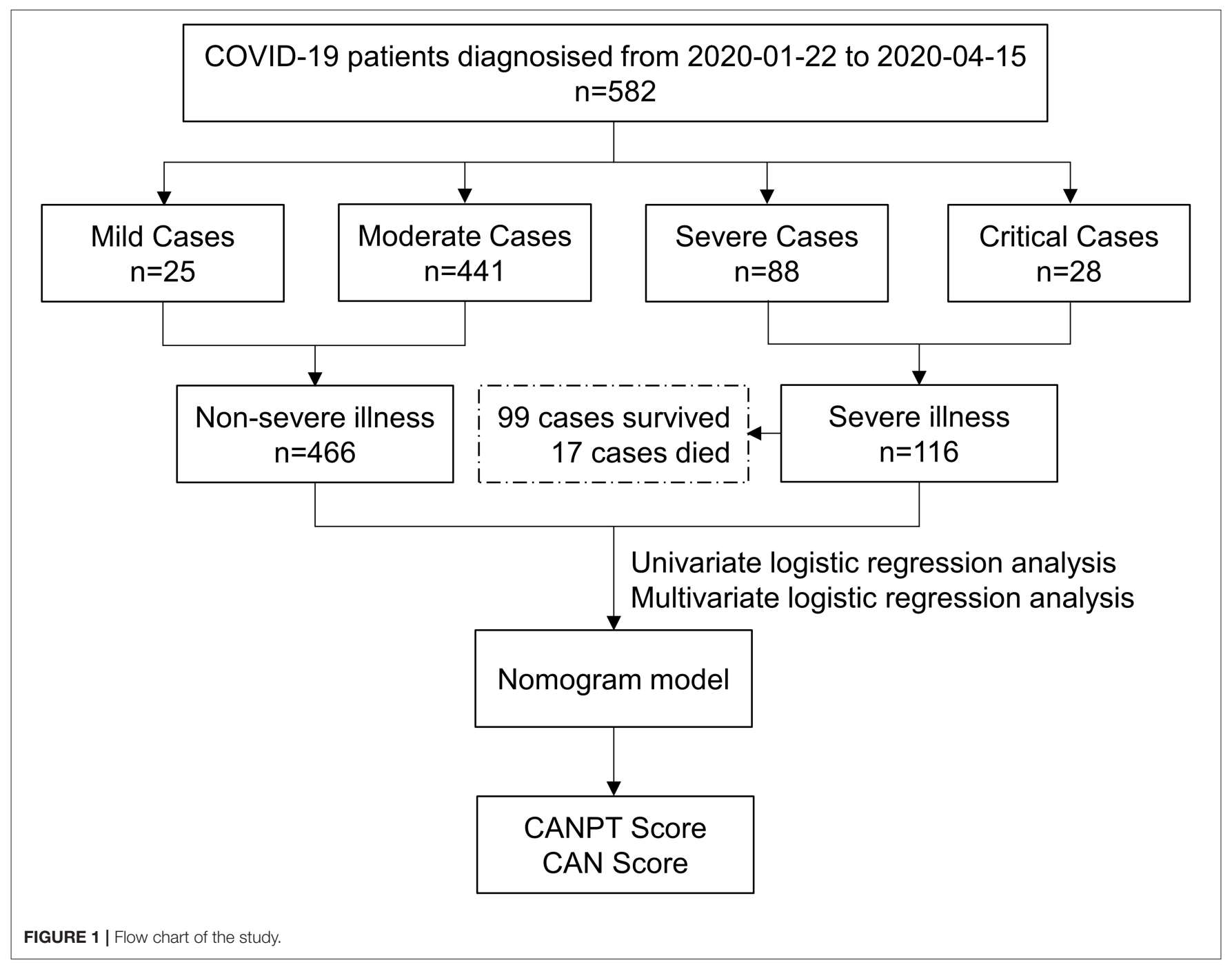

TABLE 1 | Baseline demographics and characteristics of the COVID-19 patients.

\begin{tabular}{|c|c|c|c|c|c|}
\hline Characteristics & $\begin{array}{l}\text { All patients } \\
\quad n=582\end{array}$ & $\begin{array}{l}\text { Severe disease } \\
\quad n=116\end{array}$ & $\begin{array}{l}\text { Non-severe disease } \\
\qquad n=466\end{array}$ & $t / z / \chi^{2}$ value & $P$-value \\
\hline Sex (male) & 286 (49.1\%) & 62 (53.5\%) & 224 (48.1\%) & 1.08 & 0.300 \\
\hline Heart rate, beats per minute & $86.0(78.0,97.0)$ & $88.0(80.0,101.0)$ & $85.0(78.0,96.0)$ & -1.55 & 0.121 \\
\hline Respiratory rate, breaths per minute & $20.0(18.0,20.0)$ & $20.0(18.0,21.0)$ & $20.0(18.0,20.0)$ & -2.16 & 0.033 \\
\hline Hypertension & $117(20.1 \%)$ & $43(37.1 \%)$ & 74 (15.9\%) & 25.96 & $<0.001$ \\
\hline Diabetes & $54(9.3 \%)$ & $24(20.7 \%)$ & $30(6.4 \%)$ & 22.41 & $<0.001$ \\
\hline Malignant tumor & $13(2.2 \%)$ & $7(6.0 \%)$ & $6(1.3 \%)$ & 9.58 & 0.002 \\
\hline \multicolumn{6}{|l|}{ Clinical symptoms } \\
\hline Fever & 431 (74.1\%) & $93(80.2 \%)$ & $338(72.5 \%)$ & 2.82 & 0.093 \\
\hline
\end{tabular}

MAP, mean arterial pressure. 
TABLE 2 | Baseline blood and biochemical indices of the COVID-19 patients at baseline.

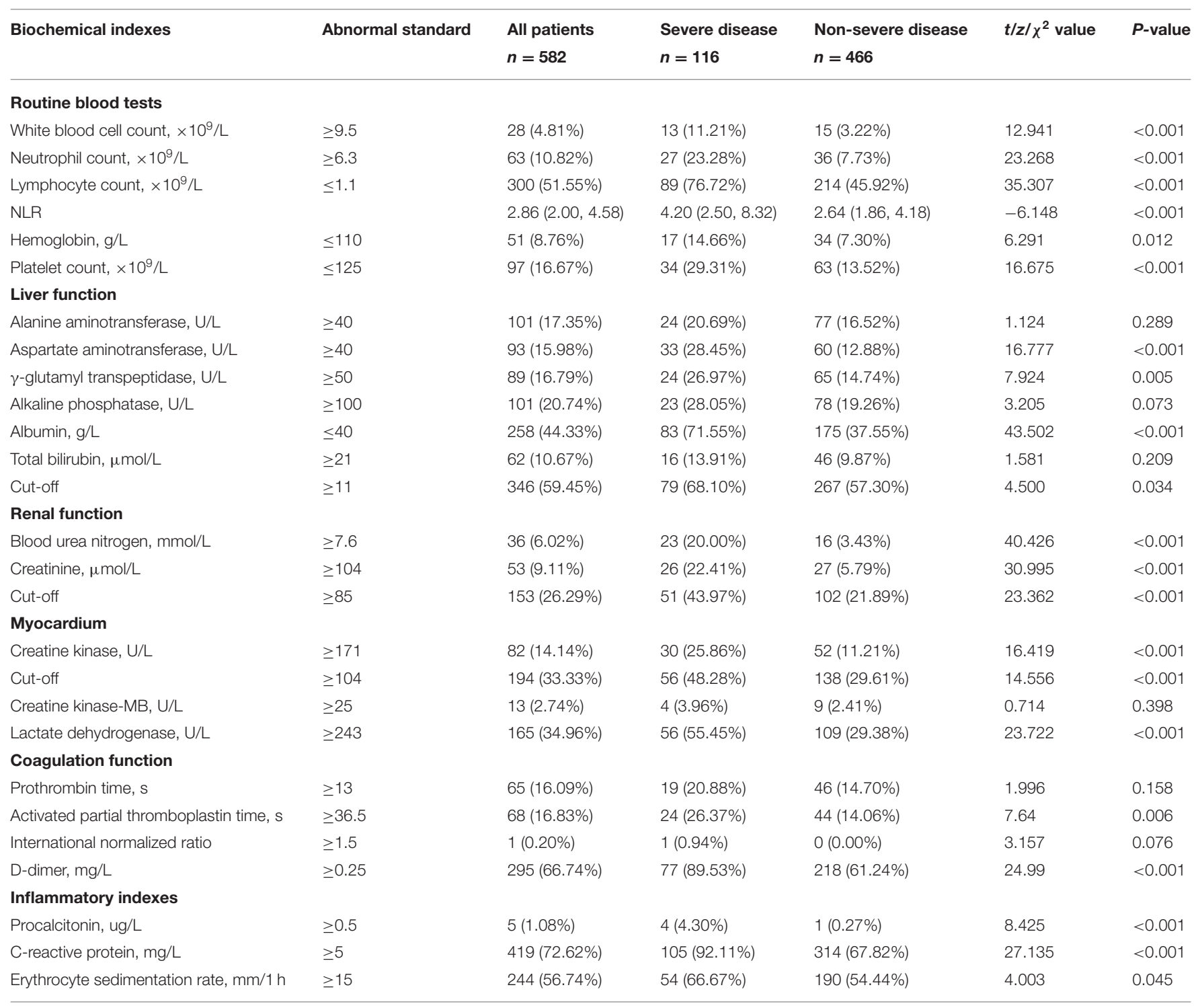

Missing data of baseline blood and biochemical indices: variables with missing values exceeding 10\% include: procalcitonin (118 cases), erythrocyte sedimentation rate (152 cases), alkaline phosphatase (95 cases), creatine kinase-MB (108 cases), lactate dehydrogenase (110 cases), prothrombin time and activated partial thromboplastin time (178 cases), and D-dimer (140 cases).

NLR, neutrophil-to-lymphocyte ratio.

(Alb) level was more common in the patients with severe disease than those with non-severe disease $(71.55 v s .37 .55 \%, P<0.001)$. There were larger proportions of patients with abnormal levels of blood urea nitrogen (BUN), creatinine (Cr), creatine kinase (CK), LDH, D-dimer, PCT, and CRP; activated partial thromboplastin time (APTT); and erythrocyte sedimentation rate (ESR) in the group with severe disease than in the group with non-severe disease (Table 2).

\section{Independent Risk Factors for Severe COVID-19}

According to univariate logistic regression analysis, 24 variables, including age, comorbidities, fever, and $\ln (\mathrm{CRP})$, were associated with the severity of COVID-19 and included in the multivariate logistic regression analysis (forward likelihood method). A body temperature $\geq 38.5^{\circ} \mathrm{C}, \ln (\mathrm{NLR}), \ln$ (PLT), ln (Alb), ln (Tbil), $\ln (\mathrm{Cr})$, and $\ln (\mathrm{CK})$ were independent risk factors for severe COVID-19. The risk of severe illness in the patients with a body temperature $\geq 38.5^{\circ} \mathrm{C}$ was $2.37(95 \% \mathrm{CI}, 1.39,4.03)$ times that in the patients with a body temperature $<38.5^{\circ} \mathrm{C}$. Comorbidities and high values of $\ln (\mathrm{NLR}), \ln$ (Tbil), ln (Cr), and $\ln (\mathrm{CK})$, and low values of $\ln$ (PLT) and $\ln (\mathrm{Alb})$ were associated with an increased risk of severe COVID-19 (Table 3).

Predictive Nomogram for Severe COVID-19 Based on the aforementioned eight variables, a nomogram model was established to predict the risk of severe COVID-19 (Figure 2) with a prediction accuracy of $85.9 \%$, a leave-one-out cross 
TABLE 3 | Risk factors for severe COVID-19 and mortality.

\begin{tabular}{|c|c|c|c|c|}
\hline Variable & $\begin{array}{l}\text { Crude OR } \\
(95 \% \mathrm{Cl})\end{array}$ & $\begin{array}{c}\text { Adjusted OR } \\
(95 \% \mathrm{Cl})\end{array}$ & $\begin{array}{c}P \text {-value } \\
\text { (Wald's test) }\end{array}$ & $\begin{array}{l}P \text {-value } \\
\text { (LR-test) }\end{array}$ \\
\hline \multicolumn{5}{|c|}{ Risk factors for severe COVID-19 } \\
\hline Comorbidity & $4.48(2.93,6.86)$ & $2.69(1.61,4.49)$ & $<0.001$ & $<0.001$ \\
\hline $\begin{array}{l}\text { Temperature } \\
\geq 38.5^{\circ} \mathrm{C}\end{array}$ & $.46(1.6,3.77)$ & $2.37(1.39,4.03)$ & 0.002 & .002 \\
\hline In (NLR) & $2.75(2.04,3.71)$ & $1.64(1.13,2.37)$ & 0.009 & 0.008 \\
\hline $\ln (P L T)$ & $0.27(0.15,0.48)$ & $0.45(0.23,0.9)$ & 0.024 & 0.023 \\
\hline In (Alb) & $0.00(0.00,0.00)$ & $0.00(0.00,0.02)$ & $<0.001$ & $<0.001$ \\
\hline In (Tbil) & $2.04(1.29,3.21)$ & $1.92(1.04,3.52)$ & 0.036 & 0.032 \\
\hline $\ln (\mathrm{Cr})$ & $3.54(1.96,6.4)$ & $2.68(1.39,5.17)$ & 0.003 & 0.001 \\
\hline $\ln (\mathrm{CK})$ & $1.86(1.4,2.48)$ & $1.44(1.03,2.02)$ & 0.034 & 0.034 \\
\hline \multicolumn{5}{|c|}{ Risk factors for mortality } \\
\hline In (NLR) & $3.78(2.17,6.60)$ & $2.67(1.27,5.62)$ & $<0.001$ & 0.01 \\
\hline $\ln ($ Alb) & $0.01(0.00,0.01)$ & $0.01(0.00,0.05)$ & $<0.001$ & $<0.001$ \\
\hline $\ln (\mathrm{Cr})$ & $\begin{array}{c}7.28(3.06 \\
17.31)\end{array}$ & $\begin{array}{c}7.23(2.89, \\
18.10)\end{array}$ & $<0.001$ & $<0.001$ \\
\hline $\ln (C K)$ & $3.29(1.93,5.61)$ & $3.00(1.60,5.61)$ & $<0.001$ & $<0.001$ \\
\hline
\end{tabular}

NLR, neutrophil-to-lymphocyte ratio; PLT, platelet; Alb, albumin; Tbil, total bilirubin; Cr, creatinine; CK, creatine kinase; In, natural logarithm.

validation accuracy of $81.6 \%$, an AUROC of 0.858 (95\% CI, $0.823-0.893)$, and a Hosmer-Lemeshow test $P$-value of 0.237 .

\section{CANPT Score: A Novel Scoring Model for the Prediction of the Risk of Severe COVID-19}

For convenience in clinical use, a novel scoring model was constructed based on the nomogram model and named the CANPT score, with scores ranging from 8 to 20 (Table 4). The AUROC of the CANPT score was 0.841 (95\% CI, 0.804, 0.879), with a positive predictive value of $35.49 \%$ (95\% CI, 30.23\%, $41.13 \%$ ), and a negative predictive value of $95.85 \%$ (95\% CI, $92.81 \%, 97.68 \%$ ) when 12 was used as the first cut-off value, and a positive predictive value of $69.64 \%$ (95\% CI, 56.6\%, 80.16\%) and a negative predictive value of $85.36 \%$ (95\% CI, $82.07 \%, 88.14 \%$ ) when 16 was used as the second cut-off value (Table 5). In this study, $12 / 289$ patients with a CANPT score $<12$ developed severe disease; 65/237 patients with a CANPT score $\geq 12$ and $<16$ developed severe disease; and 39/56 patients with a CANPT score $\geq 16$ developed severe disease. The actual incidence of severe disease in the COVID-19 patients with CANPT scores $<12, \geq 12$ and $<16$, and $\geq 16$ were $4.15,27.43$, and $69.64 \%$, respectively. Thus, with cut-off values of 12 and 16, COVID-19 patients could be classified into low-risk, medium-risk, and high-risk groups with corresponding risks of developing severe COVID-19 of $<5$, 30 , and $70 \%$, respectively.

In this study, the CALL scores were calculated for 472 COVID-19 patients who had measurements of serum LDH levels on admission, and the predictive efficacies of the CALL score and the NLR were verified among these patients and compared with the efficacy of the CANPT score. The results showed that the AUROCs of the CANPT score, CALL score, and NLR were 0.835
(95\% CI, 0.794, 0.876), 0.795 (95\% CI, 0.747, 0.844), and 0.669 (95\% CI, 0.607, 0.730), respectively. The predictive performance of the CANPT score was better than that of the CALL score and NLR (Figure 3).

\section{Model for the Prediction of the Risk of Mortality in COVID-19 Patients}

Multivariate logistic regression analysis showed that the NLR and the levels of Alb, Cr, and CK were independent risk factors for mortality in patients with COVID-19 (Table 3). The CAN score was established to predict the risk of mortality in patients with COVID-19. The CAN score ranged from 4 to 19 (Table 4), with a prediction accuracy of $97.3 \%$, a leave-one-out cross validation accuracy of $96.2 \%$, an AUROC of 0.903 (95\% CI, 0.832, 0.974), and a Hosmer-Lemeshow test $P$-value of 0.173 . The cut-off value was determined by maximizing the Youden index at 15 points, with a sensitivity of $76.47 \%$ (95\% CI, 52.23\%, 90.95\%), a specificity of $93.63 \%$ (95\% CI, $91.28 \%, 95.38 \%)$, a positive predictive value of $26.53 \%$ (95\% CI, $16.10 \%, 40.37 \%)$, a negative predictive value of $99.25 \%$ (95\% CI, 98.01\%, 99.78\%), a positive likelihood ratio of 12.00 (95\% CI, 11.12, 12.95), and a negative likelihood ratio of 0.25 (95\% CI, 0.21, 0.29). In this study, 49/582 patients with COVID-19 had a CAN score $\geq 15$; of these patients, 13 died. The actual mortality rates were $26.53 \%$ in the patients with a CAN score $\geq 15$ and only $0.75 \%$ in those with a CAN score $<15$. Among the patients with severe disease, the actual mortality rate was $43.33 \%$ in the patients with a CAN score $\geq 15$.

\section{DISCUSSION}

We enrolled 582 COVID-19 pneumonia patients in this study from four hospitals in three provinces, $74.57 \%$ of whom were from Hubei Province, and $25.43 \%$ of whom were from outside Hubei Province; thus, we reduced patient selection bias. Previous studies have shown that COVID-19 is a systemic disease with damage occurring not only in the lungs but also in many other systems, including the circulatory, cardiovascular, renal, gastrointestinal, endocrine, nervous, and integumentary systems $(23,24)$. In this study, some patients with COVID-19 had increased WBC and NE counts; increased ESRs; increased APTTs; increased levels of D-dimer, PCT, CRP, AST, GGT, BUN, Cr, CK, and LDH; decreased levels of HGB and Alb; and decreased PLT counts, further confirming the presence of multisystem damage in COVID-19 patients. Moreover, the incidence and degree of abnormalities in the above indicators in the patients with severe disease were significantly higher than those in the patients with non-severe disease, and the number of damaged systems and degree of damage were related to the severity of COVID-19. In this study, comorbidities, a body temperature $\geq 38.5^{\circ} \mathrm{C}$, ln (NLR), ln (PLT), ln (Alb), ln (Tbil), $\ln (\mathrm{Cr})$, and $\ln (\mathrm{CK})$ were found to be independent risk factors for severe COVID-19, and the CANPT score comprehensively reflected the presence and degree of damage to the immune system, circulatory system, liver, kidneys, and heart in the patients with COVID-19, thereby accurately predicting the risk of severe disease. 


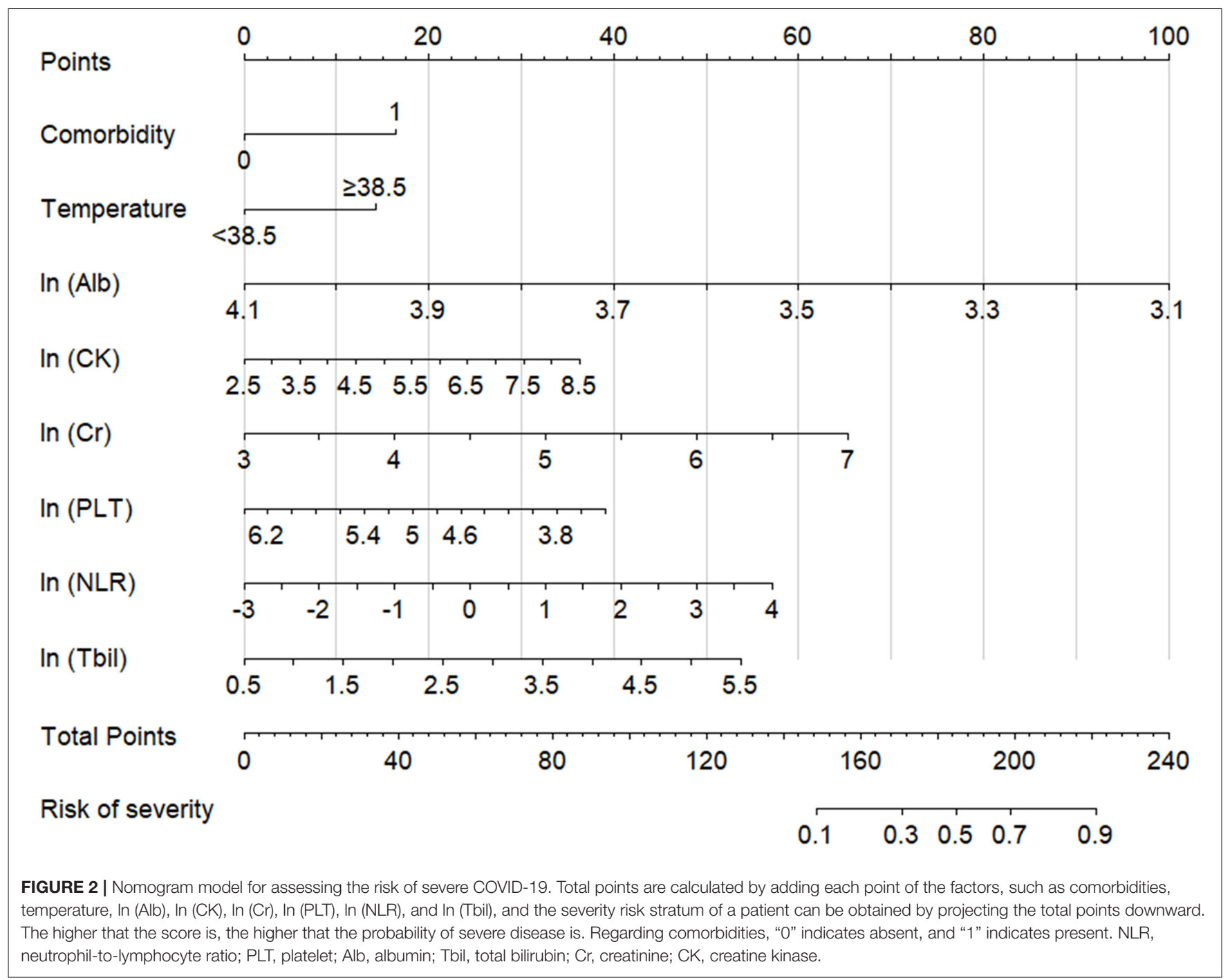

Current studies have confirmed that patients with severe COVID-19 develop SARS-CoV-2-related cytokine storms and systemic inflammatory response syndrome (SIRS) (27-29). SIRS often leads to dysfunction in the lungs, kidneys, liver, heart, etc., and even multiple organ failure syndrome (MOFS). Angiotensin converting enzyme 2 (ACE2) and transmembrane protease serine 2 (TMPRSS2) are the receptors by which SARS-CoV-2 invades cells. In addition to the respiratory system, organs and tissues, such as the kidneys, heart, and bile duct epithelium, express ACE2/TMPRSS2 and therefore are potential target organs of SARS-CoV-2 that can be directly damaged $(30,31)$. Acute kidney injury (AKI) and myocardial injury have been observed in patients with critical COVID-19 (32-34). Therefore, organs, such as the kidneys, liver, and heart, can be affected by both direct damage from SARS-CoV-2 and indirect damage mediated by SIRS. Indicators of the function of these organs, such as Alb, Tbil, and $\mathrm{Cr}$, were given relatively greater weight in the CANPT score to reflect that the degree of organ dysfunction plays an important role in the progression and severity of COVID-19.
Previous studies have shown that AKI is a common complication in patients with COVID-19 and that patients with kidney disease have a significantly higher risk of in-hospital mortality (35). Kidney biopsies from 17 patients with COVID19 complicated with kidney injury did not show SARS-CoV-2 in the kidney tissue, suggesting that kidney injury in COVID-19 patients is mainly caused by SARS-CoV-2-associated SIRS, rather than direct renal damage caused by SARS-CoV-2 (36). A previous study found that the level of $\mathrm{Cr}$ is an independent risk factor for severe COVID-19; thus, $\mathrm{Cr}$ was given relatively greater weight in the CANPT score. Therefore, CANPT score could accurately reflect the extent of renal damage in COVID-19 patients early on admission.

Recent studies have shown that almost all hospitalized patients with COVID-19 have elevated levels of serum CK and $\mathrm{LDH}$ (28, 37, 38). Autopsies of patients who died of COVID19 showed cardiomyocyte necrosis and monocyte infiltration (26). Persistently elevated CK indicates the occurrence and progression of myocardial injury in patients with COVID-19. 
TABLE 4 | Calculation of the CANPT and CAN scores.

\begin{tabular}{|c|c|c|c|}
\hline Variable & Adjusted OR (95\% Cl) & $P$-value (Wald's test) & Points \\
\hline \multicolumn{4}{|c|}{ CANPT score } \\
\hline \multicolumn{4}{|c|}{ Comorbidities } \\
\hline Present & $3.24(1.98,5.29)$ & $<0.001$ & 3 \\
\hline Absent & & & 1 \\
\hline \multicolumn{4}{|c|}{ Highest temperature } \\
\hline$\geq 38.5^{\circ} \mathrm{C}$ & $2.18(1.30,3.65)$ & 0.003 & 2 \\
\hline$<38.5^{\circ} \mathrm{C}$ & & & 1 \\
\hline \multicolumn{4}{|l|}{ NLR } \\
\hline$\geq 3.7$ & $1.90(1.17,3.11)$ & 0.01 & 2 \\
\hline$<3.7$ & & & 1 \\
\hline \multicolumn{4}{|l|}{ PLT } \\
\hline$\geq 155$ & & & 1 \\
\hline$<155$ & $1.95(1.19,3.18)$ & 0.008 & 2 \\
\hline \multicolumn{4}{|l|}{ Alb } \\
\hline$\geq 38$ & & & 1 \\
\hline$<38$ & $4.14(2.49,6.88)$ & $<0.001$ & 4 \\
\hline \multicolumn{4}{|l|}{ Tbil } \\
\hline$\geq 11$ & $1.37(0.82,2.28)$ & 0.229 & 2 \\
\hline$<11$ & & & 1 \\
\hline \multicolumn{4}{|l|}{$\mathrm{Cr}$} \\
\hline$\geq 85$ & $3.13(1.86,5.29)$ & $<0.001$ & 3 \\
\hline$<85$ & & & 1 \\
\hline \multicolumn{4}{|l|}{ CK } \\
\hline$\geq 104$ & $1.69(1.03,2.76)$ & 0.036 & 2 \\
\hline$<104$ & & & 1 \\
\hline \multicolumn{4}{|c|}{ CAN score } \\
\hline \multicolumn{4}{|l|}{ NLR } \\
\hline$\geq 7$ & $4.92(1.51,16.10)$ & 0.008 & 5 \\
\hline$<7$ & & & 1 \\
\hline \multicolumn{4}{|l|}{ Alb } \\
\hline$\geq 38$ & & & 1 \\
\hline$<38$ & $2.50(1.47,4.24)$ & 0.001 & 2 \\
\hline \multicolumn{4}{|l|}{$\mathrm{Cr}$} \\
\hline$\geq 80$ & $8.62(2.24,33.24)$ & 0.002 & 8 \\
\hline$<80$ & & & 1 \\
\hline \multicolumn{4}{|l|}{ CK } \\
\hline$\geq 106$ & $4.34(1.32,14.28)$ & 0.016 & 4 \\
\hline$<106$ & & & 1 \\
\hline
\end{tabular}

NLR, neutrophil-to-lymphocyte ratio; PLT, platelet; Alb, albumin; Tbil, total bilirubin; Cr, creatinine; CK, creatine kinase.

CK is a prognostic marker for severe COVID-19, and the cutoff value is less than the upper limit of normal (ULN) (39). In this study, elevated CK levels were found in $14.14 \%$ of the patients with COVID-19 and was more common in patients with severe disease than those with non-severe disease (25.86 vs. $11.21 \%, P<$ $0.001)$. CK was given a weight of 2 in the CANPT score, reflecting myocardial damage in patients with COVID-19.

The activation of the coagulation system is very common in inflammatory and anti-inflammatory reactions and readily leads to diffuse intravascular coagulation (DIC), which plays an important role in the occurrence and development of organ
TABLE 5 | Accuracy of the CANPT score in estimating the risk of disease progression.

\begin{tabular}{l} 
Variable \\
\hline AUROC \\
Cut-off value (95\% CI) \\
Sensitivity, \% \\
Specificity, \% \\
Positive predictive value, \% \\
Negative predictive value, \% \\
Positive likelihood ratio \\
Negative likelihood ratio \\
Cut-off value (95\% CI) \\
Sensitivity, \% \\
Specificity, \% \\
Positive predictive value, \% \\
Negative predictive value, \% \\
Positive likelihood ratio \\
Negative likelihood ratio
\end{tabular}

Enrolled patients $(n=582)$

$0.841(0.804,0.879)$

12

$89.66(82.65,94.12)$

$59.44(54.92,63.81)$

$35.49(30.23,41.13)$

95.85 (92.81, 97.68)

$2.21(2.12,2.31)$

$0.17(0.16,0.19)$

16

$33.62(25.66,42.64)$

$96.35(94.2,97.75)$

69.64 (56.6, 80.16)

$85.36(82.07,88.14)$

$9.22(8.69,9.78)$

$0.689(0.671,0.706)$

AUROC, area under the receiver operating characteristic curve; Cl, confidence interval.

damage (40). Abnormal coagulation function can be observed in patients with COVID-19, and almost all patients with severe COVID-19 have coagulation disorders. Several studies have shown that abnormal coagulation parameters are closely related to a poor prognosis of COVID-19 (41-46), especially D-dimer, which was not included in the modeling analysis due to the missing value of $\mathrm{D}$-dimer being $>10 \%$. Previous studies have reported that the PLT count can be used as a marker of the progression of COVID-19 (47-51). In this study, a reduced PLT count was a risk factor for severe COVID19 , consistent with the results of previous studies. On the one hand, the reduced PLT count in patients with COVID-19 is due to the massive consumption of PLTs in the DIC process; autopsies of patients who died from COVID-19 showed that microthrombi formed in the pulmonary capillaries (26). On the other hand, SARS-CoV-2 can also directly infect bone marrow components, causing hematopoietic abnormalities or triggering an autoimmune response to blood cells $(52,53)$, further leading to a reduction in the PLT count. Therefore, the decrease in the PLT count reflects abnormal coagulation function, even DIC, in patients with COVID-19.

The presence of comorbidities is also an independent risk factor for severe COVID-19. Patients with hypertension, diabetes, structural lung disease, chronic kidney disease, etc., are more likely to develop ARDS and multiple organ dysfunction syndrome (MODS) in response to SIRS because of preexisting organ structural abnormalities and/or dysfunction (54-57). Due to the high levels of ACE2 expression on the surface, vascular endothelial cells may suffer from direct damage by SARS-CoV-2 and indirect damage due to SARS-CoV-2-associated SIRS. Patients with preexisting vascular endothelial cell damage due to diabetes, hypertension, or chronic kidney disease are predisposed to experiencing more damage to vascular endothelial cells by SARS-CoV-2, which could play a key role in the development of MOFS and severe disease (58). 


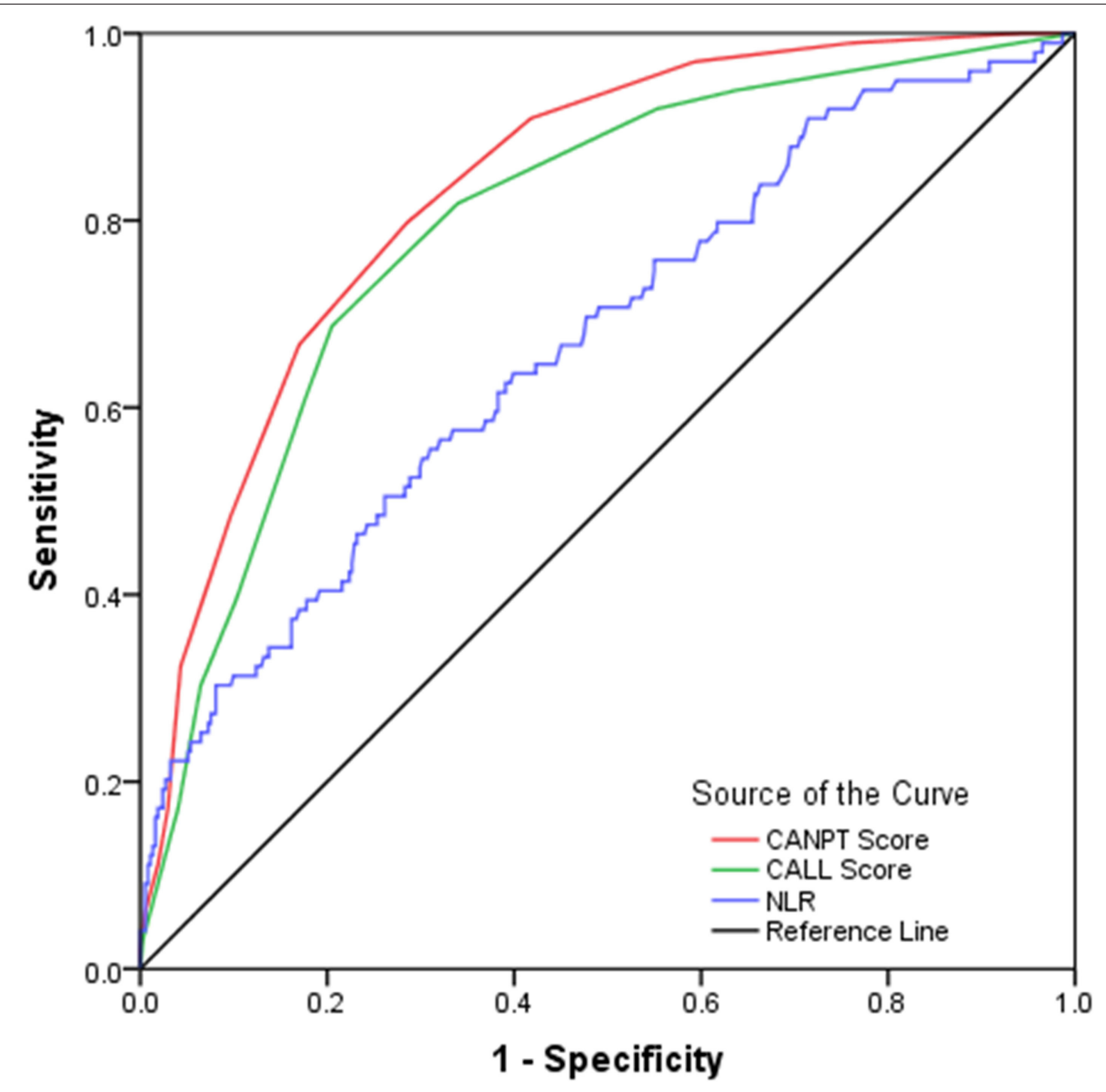

FIGURE 3 | ROC curves of the CANPT score, CALL score, and the NLR. The area under the receiver operating characteristic curves of the CANPT score, CALL score, and the NLR were 0.835 (95\% Cl, 0.794, 0.876), 0.795 (95\% Cl, 0.747, 0.844), and 0.669 (95\% Cl, 0.607, 0.730), respectively. ROC, receiver operating characteristic curve; $\mathrm{Cl}$, confidence interval; NLR, neutrophil-to-lymphocyte ratio.

In this study, a high NLR was a risk factor for severe disease in patients with COVID-19, consistent with the results of previous studies $(10,21,59)$. The elevation in the NLR is related to immune disorders in patients with COVID-19. The invasion of SARS-CoV-2 triggers cytokine storms and SIRS in the body, resulting in an increased NE count. The LY count was found to be significantly reduced in peripheral blood from patients with COVID-19 due to recruitment and translocation in the local inflammatory system, which is especially pronounced in patients with severe disease (60). Another study found that SARSCoV-2 could promote $\mathrm{T}$ lymphocyte apoptosis by activating the STAT1/IRF3 pathway (61). Thus, an elevated NE count and a reduced LY count in patients with COVID-19 lead to an increase in the NLR, reflecting the degree of the immune response and SIRS in patients with COVID-19.

Fever is a common symptom in patients with COVID-19. The immune response to SARS-CoV2 leads to systemic inflammation and even SIRS, with the consequent release of endogenous pyrogens, and the severity of fever represents the severity of SIRS (62). In this study, a body temperature $\geq 38.5^{\circ} \mathrm{C}$ was found to be more common in the group with severe disease than in the group with non-severe disease and was an independent risk factor for severe COVID-19.

ARDS is a clinical characteristic of severe COVID-19 and occurs in more than $71.2 \%$ of patients with severe COVID19 in the ICU (63). The Murray score is used to evaluate the severity of acute lung injury and the risk of ARDS. The higher the Murray score, the more severe the acute lung injury, and the higher the risk of ARDS (64). Previous studies have shown that the Alb level in patients with COVID-19 is negatively correlated with the SARS-CoV-2 load and Murray score, and the higher the SARS-CoV-2 load in patients, the more critical the patient's condition (65). Recent studies have found that a low Alb level is an independent risk factor for disease progression in patients with COVID-19 (51, 66-68). In this study, a low Alb level was an important predictor of severe disease in patients with COVID19 and was given a weight of 4 in the CANPT score, further validating previous research.

There have been reports of severe liver damage in patients with severe COVID-19 (69-71). Chai et al. suggested that liver injury in patients with COVID-19 could be caused by SARS-CoV-2-mediated injury in bile duct cells (72). However, 
SARS-CoV-2 has not been found in bile duct cells from patients who died of COVID-19, suggesting that liver damage in patients with COVID-19 is mediated by the SARS-CoV-2-associated cytokine storm and SIRS (73). Elevated ALT and AST levels suggest liver cell damage, while an elevated Tbil level indicates hepatocyte necrosis after the exclusion of bile duct obstruction or hemolysis. Several studies have found that an elevated Tbil level is significantly correlated with adverse outcomes of COVID$19(69,70,74,75)$; Tbil, which was given a weight of 2 in the CANPT score, reflects the severity of liver injury in patients with COVID-19.

Interestingly, the cut-off values of $\mathrm{CK}, \mathrm{Cr}$, and Tbil determined in this study were markedly lower than their ULNs. The rationale for CK, Cr, and Tbil levels being elevated is high risk for severe COVID-19, but the cut-off chosen for the CANPT score is lower than the ULN can be explained for the following reasons: (1) patients with higher values of $\mathrm{CK}, \mathrm{Cr}$, and Tbil than the cut-off levels could at high risk for according organ damage; and (2) higher values of $\mathrm{CK}, \mathrm{Cr}$, and Tbil may be resulted from an increase from much lower baseline levels due to organ injury; i.e., a significant increase in levels of $\mathrm{CK}, \mathrm{Cr}$, and Tbil could be important indicators of organ injury. The report from Qin et al. showed similar results in COVID-19 patients. In this study, cut-off values for high-sensitivity cardiac troponin I (hs-cTnI), creatine phosphokinase-MB (CK-MB), CK, and myoglobin (MYO) equivalent to $\sim 49 \%$ ULN and a cut-off value of N-terminal pro-brain natriuretic peptide (NT-proBNP) equivalent of $\sim 18.9 \%$ ULN were established for the prediction of adverse outcomes in COVID-19 patients, and patients with higher hs-cTnI, CK-MB, CK, MYO, and (NT-pro) BNP levels than the cut-off values were correlated with increased risk of death (39). Therefore, COVID-19 patients with CK, Cr, and Tbil levels higher than the cut-off values determined in this study, although within normal ranges, would still be at high risk for adverse outcomes and require more attention. Further, CANPT/CAN scoring could be helpful in identifying COVID-19 patients who are at high risk for severe disease.

In this study, $<5 \%$ of the COVID- 19 patients with a CANPT score $<12$ developed severe disease. Thus, the patients with a CANPT score $<12$ were considered low-risk patients, and the recommendation is to place these low-risk patients in a mobile cabin hospital or have them isolate at home with general symptomatic treatment with oral medication. In total, $27.43 \%$ of the patients with a CANPT score $\geq 12$ and $<16$ developed severe disease and were considered at intermediate risk; therefore, people with a score within this range should be admitted to an isolation ward for respiratory monitoring and receive antiviral, anti-inflammatory, and symptomatic treatment. Nearly $70 \%$ of the patients with a CANPT score $\geq 16$ developed severe disease. These patients should be considered at a high risk and should be transferred to an isolation ICU to receive comprehensive antiviral and symptomatic supportive treatment and respiratory support.

When the CAN score was used to predict the risk of mortality, only $0.75 \%$ of the patients with a CAN score $<15$ died, while among those with a CAN score $\geq 15,26.53 \%$ of all patients, and $43.33 \%$ of the patients with severe disease eventually died. Thus, the CAN score could be used to identify patients who are at a high risk for mortality. Patients with a CAN score $<15$ are relatively safe, while those with a CAN score $\geq 15$ are at a high risk for mortality regardless of whether they have severe disease and should be treated in the ICU.

The CALL score, which considers comorbidities, the LY count, age, and the $\mathrm{LDH}$ level, has been reported to have good predictive efficacy and is convenient for use in clinical practice (20). Studies have also reported that the NLR is an independent predictor of poor prognosis in patients with COVID-19 $(21,22)$. In this study, the CANPT score was compared with the CALL score and the NLR. The predictive performance of the CANPT score was significantly superior to that of the CALL score and the NLR with a larger AUROC.

The use of comorbidities and routine indicators, including body temperature, the NLR, the PLT count, and the levels of Alb, Tbil, Cr, and CK, renders the CANPT and CAN scores easy to calculate, and these scores are efficient in predicting the risk of severe illness and death in patients with COVID-19. These scores could be used to help clinicians to identify patients at high risk for poor outcomes or mortality soon after admission. Providing intensive care to the small proportions of patients who at high risk could improve their outcomes and reduce the mortality rate, and the rational allocation of limited medical staff and equipment could alleviate the serious shortages of medical resources. There were some limitations of this study. First, the practice for identification of severe and critically ill COVID-19 might differ in different hospitals, which could bias the results of the analysis. Second, this study only included Chinese patients; thus, the performance of the CANPT score and CAN score in patients of other ethnicities must be validated. Third, only 17 of the 582 COVID-19 patients included in this study died; therefore, the CAN model might not be sufficiently accurate to predict the risk of death in COVID-19 patients. Finally, this study was a retrospective study with a sample size of 582 without external validation, and the CANPT and CAN scores must be further validated in a large sample of patients from different regions.

The CANPT and CAN scores can be used to identify patients with COVID-19 who are at a high risk for severe disease or death soon after admission, guiding patient management and the rational allocation of limited medical resources based on patient risk stratification.

\section{DATA AVAILABILITY STATEMENT}

The data analyzed in this study is subject to the following licenses/restrictions: Clinical data were retrieved from the medical records databases of Shiyan Taihe Hospital, Ankang Central Hospital, Ningbo Hwamei Hospital, and Yichang Central People's Hospital. Requests to access these datasets should be directed to Yuanyuan Chen, cyy15871089714@163.com.

\section{ETHICS STATEMENT}

The studies involving human participants were reviewed and approved by the Medical Ethics Committee of Shiyan Taihe Hospital. The approval number is 2020KS018. Written 
informed consent for participation was not required for this study in accordance with the national legislation and the institutional requirements.

\section{AUTHOR CONTRIBUTIONS}

YC and ZM designed and coordinated the research, contributed to the statistical analysis, and interpretation and the writing of the manuscript. ZM reviewed and edited the manuscript. JZ guided the data analysis. YC, HY, XZ, HH, ZJ, and SL collected the data. All authors contributed to and approved the submitted version of this manuscript.

\section{REFERENCES}

1. Zhou P, Yang XL, Wang XG, Hu B, Zhang L, Zhang W, et al. A pneumonia outbreak associated with a new coronavirus of probable bat origin. Nature. (2020) 579:270-3. doi: 10.1038/s41586-020-2012-7

2. Zhu N, Zhang D, Wang W, Li X, Yang B, Song J, et al. A novel coronavirus from patients with pneumonia in China, 2019. N Engl J Med. (2020) 382:72733. doi: 10.1056/NEJMoa2001017

3. Lu R, Zhao $\mathrm{X}$, Li J, Niu P, Yang $\mathrm{B}$, Wu H, et al. Genomic characterisation and epidemiology of 2019 novel coronavirus: implications for virus origins and receptor binding. Lancet. (2020) 395:565-74. doi: 10.1016/S0140-6736(20)30251-8

4. Wu JT, Leung K, Leung GM. Nowcasting and forecasting the potential domestic and international spread of the 2019-nCoV outbreak originating in Wuhan, China: a modelling study. Lancet. (2020) 395:689-97. doi: 10.1016/S0140-6736(20)30260-9

5. Li Q, Guan X, Wu P, Wang X, Zhou L, Tong Y, et al. Early transmission dynamics in Wuhan, China, of novel coronavirus-infected pneumonia. NEngl J Med. (2020) 382:1199-207. doi: 10.1056/Nejmoa2001316

6. World Health Organization. Coronavirus disease 2019 (COVID-19) Situation report. Available online at: https://www.who.int/emergencies/diseases/novelcoronavirus-2019/situation-reports (accessed March 01, 2020).

7. Zhang Y, Chen Y, Meng Z. Immunomodulation for severe COVID19 pneumonia: the state of the art. Front Immunol. (2020) 11:577442. doi: 10.3389/fimmu.2020.577442

8. Wynants L, Van Calster B, Collins GS, Riley RD, Heinze G, Schuit E, et al. Prediction models for diagnosis and prognosis of covid-19: systematic review and critical appraisal. BMJ. (2020) 369:m1328. doi: 10.1136/bmj.m1328

9. Yuan M, Yin W, Tao Z, Tan W, Hu Y. Association of radiologic findings with mortality of patients infected with 2019 novel coronavirus in Wuhan, China. PLoS ONE. (2020) 15:e0230548. doi: 10.1371/journal.pone.0230548

10. Cheng B, Hu J, Zuo X, Chen J, Li X, Chen Y, et al. Predictors of progression from moderate to severe coronavirus disease 2019: a retrospective cohort. Clin Microbiol Infect. (2020) 26:1400-5. doi: 10.1016/j.cmi.2020.06.033

11. Ye W, Chen G, Li X, Lan X, Ji C, Hou M, et al. Dynamic changes of Ddimer and neutrophil-lymphocyte count ratio as prognostic biomarkers in COVID-19. Respir Res. (2020) 21:169. doi: 10.1186/s12931-020-01428-7

12. Dong YM, Sun J, Li YX, Chen Q, Liu QQ, Sun Z, et al. Development and validation of a nomogram for assessing survival in patients with COVID-19 pneumonia. Clin Infect Dis. (2020) 10:ciaa963. doi: 10.1093/cid/ciaa963

13. Yu C, Lei Q, Li W, Wang X, Liu W, Fan X, et al. Clinical characteristics, associated factors, and predicting COVID-19 mortality risk: a retrospective study in Wuhan, China. Am J Prev Med. (2020) 59:168-75. doi: 10.1016/j.amepre.2020.05.002

14. Zheng Y, Xiao A, Yu X, Zhao Y, Lu Y, Li X, et al. Development and validation of a prognostic nomogram based on clinical and CT features for adverse outcome prediction in patients with COVID-19. Korean J Radiol. (2020) 21:1007. doi: 10.3348/kjr.2020. 0485

15. Cheng A, Hu L, Wang Y, Huang L, Zhao L, Zhang C, et al. Diagnostic performance of initial blood urea nitrogen combined with D-dimer levels

\section{FUNDING}

This work was supported by the National Science and Technology Major Project (2018ZX10302206 and 2018ZX10723203), the Foundation for Innovative Research Groups of Hubei Provincial Natural Science Foundation (2018CFA031), the Project of Hubei University of Medicine (FDFR201902 and 2020XGFYZR05), the Project of Science and Technology Plan of Shiyan (18K78 and 19Y27), and the Innovative Research Program for Graduates of Hubei University of Medicine (YC2019006).

for predicting in-hospital mortality in COVID-19 patients. Int J Antimicrob Agents. (2020) 56:106110. doi: 10.1016/j.ijantimicag.2020.106110

16. Wu G, Yang P, Xie Y, Woodruff HC, Rao X, Guiot J, et al. Development of a clinical decision support system for severity risk prediction and triage of COVID-19 patients at hospital admission: an international multicenter study. Eur Respiratory J. (2020) 56:2001104. doi: 10.1183/13993003.01104-2020

17. Wu Q, Wang S, Li L, Wu Q, Qian W, Hu Y, et al. Radiomics analysis of computed tomography helps predict poor prognostic outcome in COVID-19. Theranostics. (2020) 10:7231-44. doi: 10.7150/thno.46428

18. Liang W, Liang H, Ou L, Chen B, Chen A, Li C, et al. Development and validation of a clinical risk score to predict the occurrence of critical illness in hospitalized patients with COVID-19. JAMA Intern Med. (2020) 180:10819. doi: 10.1001/jamainternmed.2020.2033

19. Collins GS, van Smeden M, Riley RD. COVID-19 prediction models should adhere to methodological and reporting standards. Eur Respir J. (2020) 56:2020. doi: 10.1183/13993003.02643-2020

20. Ji D, Zhang D, Xu J, Chen Z, Yang T, Zhao P, et al. Prediction for progression risk in patients with COVID-19 pneumonia: the CALL score. Clin Infect Dis. (2020) 71:1393-9. doi: 10.1093/cid/ciaa414

21. Yang AP, Liu JP, Tao WQ, Li HM. The diagnostic and predictive role of NLR, d-NLR and PLR in COVID-19 patients. Int Immunopharmacol. (2020) 84:106504. doi: 10.1016/j.intimp.2020.106504

22. Yan X, Li F, Wang X, Yan J, Zhu F, Tang S, et al. Neutrophil to lymphocyte ratio as prognostic and predictive factor in patients with coronavirus disease 2019: a retrospective cross-sectional study. J Med Virol. (2020) 92:257381. doi: 10.1002/jmv.26061

23. Gupta A, Madhavan MV, Sehgal K, Nair N, Mahajan S, Sehrawat TS, et al. Extrapulmonary manifestations of COVID-19. Nat Med. (2020) 26:101732. doi: 10.1038/s41591-020-0968-3

24. Vabret N, Britton GJ, Gruber C, Hegde S, Kim J, Kuksin M, et al. Immunology of COVID-19: current state of the science. Immunity. (2020) 52:91041. doi: 10.1016/j.immuni.2020.05.002

25. Collins GS, Wilkinson J. Statistical issues in the development of COVID-19 prediction models. J Med Virol. (2020) 93:624-5. doi: 10.1002/jmv.26390

26. National Health Commission of the People's Republic of China. Guidance for 2019 Corona Virus Disease Prevention, Control, Diagnosis and Management. Available online at: http://books.ipmph.com/books/detail/2035540.shtml

27. Wu D, Yang XO. TH17 responses in cytokine storm of COVID-19: an emerging target of JAK2 inhibitor Fedratinib. J Microbiol Immunol Infect. (2020) 53:368-70. doi: 10.1016/j.jmii.2020.03.005

28. Guzik TJ, Mohiddin SA, Dimarco A, Patel V, Savvatis K, Marelli-Berg FM, et al. COVID-19 and the cardiovascular system: implications for risk assessment, diagnosis, and treatment options. Cardiovasc Res. (2020) 116:1666-87. doi: 10.1093/cvr/cvaa106

29. Tang Y, Liu J, Zhang D, Xu Z, Ji J, Wen C. Cytokine storm in COVID19: the current evidence and treatment strategies. Front Immunol. (2020) 11:1708. doi: 10.3389/fimmu.2020.01708

30. Hoffmann M, Kleine-Weber H, Schroeder S, Kruger N, Herrler T, Erichsen $\mathrm{S}$, et al. SARS-CoV-2 cell entry depends on ACE2 and TMPRSS2 and is blocked by a clinically proven protease inhibitor. Cell. (2020) 181:271-80 e278. doi: 10.1016/j.cell.2020.02.052 
31. Wan Y, Shang J, Graham R, Baric RS, Li F. Receptor recognition by the novel coronavirus from Wuhan: an analysis based on decade-long structural studies of SARS coronavirus. J Virol. (2020) 94:e00127-20. doi: 10.1128/JVI.00127-20

32. Mehta P, McAuley DF, Brown M, Sanchez E, Tattersall RS, Manson JJ, et al. COVID-19: consider cytokine storm syndromes and immunosuppression. Lancet. (2020) 395:1033-4. doi: 10.1016/S0140-6736(20)30628-0

33. Xu Z, Shi L, Wang Y, Zhang J, Huang L, Zhang C, et al. Pathological findings of COVID-19 associated with acute respiratory distress syndrome. Lancet Respiratory Med. (2020) 8:420-2. doi: 10.1016/S2213-2600(20)30076-X

34. Tian S, Xiong Y, Liu H, Niu L, Guo J, Liao M, et al. Pathological study of the 2019 novel coronavirus disease (COVID-19) through postmortem core biopsies. Mod Pathol. (2020) 33:1007-14. doi: 10.1038/s41379-020-0536-X

35. Cheng Y, Luo R, Wang K, Zhang M, Wang Z, Dong L, et al. Kidney disease is associated with in-hospital death of patients with COVID-19. Kidney Int. (2020) 97:829-38. doi: 10.1016/j.kint.2020.03.005

36. Kudose S, Batal I, Santoriello D, Xu K, Barasch J, Peleg Y, et al. Kidney biopsy findings in patients with COVID-19. J Am Soc Nephrol. (2020) 31:195968. doi: 10.1681/ASN.2020060802

37. Huang C, Wang Y, Li X, Ren L, Zhao J, Hu Y, et al. Clinical features of patients infected with 2019 novel coronavirus in Wuhan, China. Lancet. (2020) 395:497-506. doi: 10.1016/S0140-6736(20)30183-5

38. Lippi G, Lavie CJ, Sanchis-Gomar F. Cardiac troponin I in patients with coronavirus disease 2019 (COVID-19): evidence from a meta-analysis. Prog Cardiovasc Dis. (2020) 63:390-1. doi: 10.1016/j.pcad.2020.03.001

39. Qin JJ, Cheng X, Zhou F, Lei F, Akolkar G, Cai J, et al. Redefining cardiac biomarkers in predicting mortality of inpatients with COVID-19. Hypertension. (2020) 76:110412. doi: 10.1161/HYPERTENSIONAHA.120.15528

40. Esmon CT. Possible involvement of cytokines in diffuse intravascular coagulation and thrombosis. Baillieres Best Pract Res Clin Haematol. (1999) 12:343-59. doi: 10.1053/beha.1999.0029

41. Dolhnikoff M, Duarte-Neto AN, de Almeida Monteiro RA, da Silva LFF, de Oliveira EP, Saldiva PHN, et al. Pathological evidence of pulmonary thrombotic phenomena in severe COVID-19. J Thromb Haemost. (2020) 18:1517-9. doi: 10.1111/jth.14844

42. Lillicrap D. Disseminated intravascular coagulation in patients with 2019-nCoV pneumonia. J Thromb Haemost. (2020) 18:7867. doi: $10.1111 /$ jth.14781

43. Tang N, Li D, Wang X, Sun Z. Abnormal coagulation parameters are associated with poor prognosis in patients with novel coronavirus pneumonia. J Thromb Haemost. (2020) 18:844-7. doi: 10.1111/jth.14768

44. Giannis D, Ziogas IA, Gianni P. Coagulation disorders in coronavirus infected patients: COVID-19, SARS-CoV-1, MERS-CoV and lessons from the past. $J$ Clin Virol. (2020) 127:104362. doi: 10.1016/j.jcv.2020.104362

45. Song JC, Wang G, Zhang W, Zhang Y, Li WQ, Zhou Z, et al. Chinese expert consensus on diagnosis and treatment of coagulation dysfunction in COVID-19. Mil Med Res. (2020) 7:19. doi: 10.1186/s40779-020-00247-7

46. Ramlall V, Thangaraj PM, Meydan C, Foox J, Butler D, Kim J, et al. Immune complement and coagulation dysfunction in adverse outcomes of SARS-CoV2 infection. Nat Med. (2020) 26:1609-15. doi: 10.1038/s41591-020-1021-2

47. Henry BM, de Oliveira MHS, Benoit S, Plebani M, Lippi G. Hematologic, biochemical and immune biomarker abnormalities associated with severe illness and mortality in coronavirus disease 2019 (COVID-19): a metaanalysis. Clin Chem Lab Med. (2020) 58:1021-8. doi: 10.1515/cclm-2020-0369

48. Lippi G, Plebani M, Henry BM. Thrombocytopenia is associated with severe coronavirus disease 2019 (COVID-19) infections: a meta-analysis. Clin Chim Acta. (2020) 506:145-8. doi: 10.1016/j.cca.2020.03.022

49. Bi X, Su Z, Yan $\mathrm{H}, \mathrm{Du} J$, Wang J, Chen L, et al. Prediction of severe illness due to COVID-19 based on an analysis of initial Fibrinogen to Albumin Ratio and Platelet count. Platelets. (2020) 31:6749. doi: 10.1080/09537104.2020.1760230

50. Bao C, Tao X, Cui W, Yi B, Pan T, Young KH, et al. SARS-CoV-2 induced thrombocytopenia as an important biomarker significantly correlated with abnormal coagulation function, increased intravascular blood clot risk and mortality in COVID-19 patients. Exp Hematol Oncol. (2020) 9:16. doi: 10.1186/s40164-020-00172-4

51. Zhang K, Liu X, Shen J, Li Z, Sang Y, Wu X, et al. Clinically applicable AI system for accurate diagnosis, quantitative measurements, and prognosis of
COVID-19 pneumonia using computed tomography. Cell. (2020) 181:142333 e1411. doi: 10.1016/j.cell.2020.04.045

52. Yang M, Ng MHL, Li CK. Thrombocytopenia in patients with severe acute respiratory syndrome (review). Hematology. (2013) 10:101-5. doi: 10.1080/10245330400026170

53. Jolicoeur P, Lamontagne L. Impairment of bone marrow pre-B and B cells in MHV3 chronically-infected mice. Adv Exp Med Biol. (1995) 380:1935. doi: 10.1007/978-1-4615-1899-0_33

54. Chen Y, Gong X, Wang L, Guo J. Effects of hypertension, diabetes and coronary heart disease on COVID-19 diseases severity: a systematic review and meta-analysis. medRxiv. doi: 10.1101/2020.03.25.20043133

55. Hespanhol V, Bárbara C. Pneumonia mortality, comorbidities matter? Pulmonology. (2020) 26:123-9. doi: 10.1016/j.pulmoe.2019.10.003

56. Zou Q, Zheng S, Wang X, Liu S, Bao J, Yu F, et al. Influenza A-associated severe pneumonia in hospitalized patients: risk factors and NAI treatments. Int J Infect Dis. (2020) 92:208-13. doi: 10.1016/j.ijid.2020.01.017

57. Wang X, Fang X, Cai Z, Wu X, Gao X, Min J, et al. Comorbid chronic diseases and acute organ injuries are strongly correlated with disease severity and mortality among COVID-19 patients: a systemic review and meta-analysis. Research. (2020) 2020:2402961. doi: 10.34133/2020/2402961

58. Varga Z, Flammer AJ, Steiger P, Haberecker M, Andermatt R, Zinkernagel AS, et al. Endothelial cell infection and endotheliitis in COVID-19. Lancet. (2020) 395:1417-8. doi: 10.1016/S0140-6736(20)30937-5

59. Liu J, Liu Y, Xiang P, Pu L, Xiong H, Li C, et al. Neutrophil-to-lymphocyte ratio predicts severe illness patients with 2019 novel coronavirus in the early stage. J Transl Med. (2020) 18:206. doi: 10.1186/s12967-020-02374-0

60. Diao B, Wang C, Tan Y, Chen X, Liu Y, Ning L, et al. Reduction and functional exhaustion of T cells in patients with coronavirus disease 2019 (COVID-19). Front Immunol. (2020) 11:827. doi: 10.3389/fimmu.2020.00827

61. Zhu L, Yang P, Zhao Y, Zhuang Z, Wang Z, Song R, et al. Single-cell sequencing of peripheral mononuclear cells reveals distinct immune response landscapes of COVID-19 and influenza patients. Immunity. (2020) 53:685-96 e683. doi: 10.1016/j.immuni.2020.07.009

62. Garami A, Steiner AA, Romanovsky AA. Fever and hypothermia in systemic inflammation. Handb Clin Neurol. (2018) 157:56597. doi: 10.1016/B978-0-444-64074-1.00034-3

63. Yu Y, Xu D, Fu S, Zhang J, Yang X, Xu L, et al. Patients with COVID19 in 19 ICUs in Wuhan, China: a cross-sectional study. Crit Care. (2020) 24:219. doi: 10.1186/s13054-020-02939-x

64. Murray JF, Matthay MA, Luce JM, Flick MR. An expanded definition of the adult respiratory distress syndrome. Am Rev Respir Dis. (1988) 138:7203. doi: 10.1164/ajrccm/138.3.720

65. Liu Y, Yang Y, Zhang C, Huang F, Wang F, Yuan J, et al. Clinical and biochemical indexes from 2019-nCoV infected patients linked to viral loads and lung injury. Sci China Life Sci. (2020) 63:364-74. doi: 10.1007/s11427-020-1643-8

66. Zhang J, Wang X, Jia X, Li J, Hu K, Chen G, et al. Risk factors for disease severity, unimprovement, and mortality in COVID-19 patients in Wuhan, China. Clin Microbiol Infect. (2020) 26:767-72. doi: 10.1016/j.cmi.2020.04.012

67. de la Rica R, Borges M, Aranda M, Del Castillo A, Socias A, Payeras A, et al. Low albumin levels are associated with poorer outcomes in a case series of COVID-19 patients in Spain: a retrospective cohort study. Microorganisms. (2020) 8:94987. doi: 10.1101/2020.05.07.20094987

68. Huang W, Li C, Wang Z, Wang H, Zhou N, Jiang J, et al. Decreased serum albumin level indicates poor prognosis of COVID-19 patients: hepatic injury analysis from 2,623 hospitalized cases. Sci China Life Sci. (2020) 63:167887. doi: 10.1007/s11427-020-1733-4

69. Lei F, Liu YM, Zhou F, Qin JJ, Zhang P, Zhu L, et al. Longitudinal association between markers of liver injury and mortality in COVID-19 in China. Hepatology. (2020) 72:389-98. doi: 10.1002/hep.31301

70. Phipps MM, Barraza LH, LaSota ED, Sobieszczyk ME, Pereira MR, Zheng EX, et al. Acute liver injury in COVID-19: prevalence and association with clinical outcomes in a large U.S. Cohort. Hepatology. (2020) 72:80717. doi: 10.1002/hep.31404

71. Parohan M, Yaghoubi S, Seraji A. Liver injury is associated with severe coronavirus disease 2019 (COVID-19) infection: a systematic review and meta-analysis of retrospective studies. Hepatol Res. (2020) 50:92435. doi: $10.1111 /$ hepr.13510 
72. Xu L, Liu J, Lu M, Yang D, Zheng X. Liver injury during highly pathogenic human coronavirus infections. Liver Int. (2020) 40:9981004. doi: $10.1111 /$ liv. 14435

73. Li J, Fan JG. Characteristics and mechanism of liver injury in 2019 coronavirus disease. J Clin Transl Hepatol. (2020) 8:13-7. doi: 10.14218/JCTH.2020.00019

74. Fu Y, Zhu R, Bai T, Han P, He Q, Jing M, et al. Clinical features of patients infected with coronavirus disease 2019 with elevated liver biochemistries: a multicenter, retrospective study. Hepatology. (2020). doi: 10.1002/hep.31446. [Epub ahead of print].

75. Chen LY, Chu HK, Bai T, Tu SJ, Wei Y, Li ZL, et al. Liver damage at admission is an independent prognostic factor for COVID-19. J Digest Dis. (2020) 21:512-8. doi: 10.1111/1751-2980.12925
Conflict of Interest: The authors declare that the research was conducted in the absence of any commercial or financial relationships that could be construed as a potential conflict of interest.

Copyright (c) 2021 Chen, Zhou, Yan, Huang, Li, Jiang, Zhao and Meng. This is an open-access article distributed under the terms of the Creative Commons Attribution License (CC BY). The use, distribution or reproduction in other forums is permitted, provided the original author(s) and the copyright owner(s) are credited and that the original publication in this journal is cited, in accordance with accepted academic practice. No use, distribution or reproduction is permitted which does not comply with these terms. 